\title{
Lecture \\ Prospective memory in nonhuman primates
}

\author{
Michael J. Beran ${ }^{11}$, Bonnie M. PERdue ${ }^{2)}$ and TheOdORE A. EvanS ${ }^{11}$
}

\begin{abstract}
Prospective memory requires forming an intention that cannot be completed at the present time, remembering that intention, and then recognizing the appropriate time to execute it. We review recent work in our laboratory that has revealed prospective memory-like patterns of performance in nonhuman primates. Rhesus monkeys and capuchin monkeys were given computerized tasks in which the monkeys either had to remember a future response they could not make immediately, or they sometimes saw a particular stimulus during an ongoing task they had to remember to later indicate seeing or not seeing. Most monkeys succeeded on these tasks and even anticipated the necessary responses. Chimpanzees also showed evidence of prospective memory. They appeared to form intentions about less valuable food items that they did not want immediately but would want later, and they responded at a later time when it was possible to obtain those items. Even when the response option was embedded within an ongoing task, the chimpanzees still showed some success in remembering to carry out the prospective intention. This research indicates that nonhuman primates form intentions for future responses, maintain those intentions during a delay, and execute them at an appropriate time.
\end{abstract}

Key words : Prospective memory, Chimpanzees, Rhesus monkeys, Capuchin monkeys, Prospective cognition

\section{Prospective Memory in Nonhuman Primates}

Being able to anticipate the future, even on a timescale of seconds or minutes, frees one from being stuck "in the now" and allows for responses that are not tied solely to present stimuli (Atance \& O’Neil, 2001). In some ways, being able to anticipate the future may offer greater adaptive advantages than being able to remember the past (e.g., Klein, Robertson, \& Delton, 2010; Suddendorf

1) Language Research Center, Georgia State University

Language Research Center, Georgia State University, University Plaza, Atlanta, GA 30302, USA

2) Department of Psychology, Agnes Scott College

141 E. College Ave., Decatur, GA 30030, USA

Corresponding author: MiCHAEL J. BERAN

E-mail:mjberan@yahoo.com

J-STAGE Advance Published Date: April 22, 2015. doi: $10.2502 /$ janip.65.1.2
\& Busby, 2003; Suddendorf \& Corballis, 2007). Adult humans anticipate a wide range of future events on very long timescales (e.g., their retirement, where they might travel for their next vacation), intermediate timescales (e.g., what they want to cook for dinner, that they need to pick up milk from the store) and short timescales (e.g., to attach a file to an email message that is being written, to turn on the coffee maker as soon as they walk to the kitchen). In many cases, when they do these things, they experience a particular aspect to the anticipation of the future and this aspect has come to be of great interest in comparative cognitive science. This aspect is called mental time travel which includes projection of oneself into one's personal past, as when someone remembers or re-experiences specific events, or into a personal future, as when someone mentally imagines or pre-experiences how that future might transpire (Clayton et al., 
2008; Griffiths, Dickinson, \& Clayton, 1999; Roberts, 2002, 2007; Tulving, 2005; Zentall, 2006). For example, the anticipation of a future vacation or of retirement might involve this aspect of also "pre-experiencing" those events.

However, mental time travel is not a necessary component in studying prospective cognition (see Crystal, 2013; Shettleworth, 2007; Zentall, 2006) or for studying when and how animals (or humans) use what they know about their potential future to guide their present behavior. For example, anticipating the need to add an attachment to an email, as soon as one finishes writing the email, likely does not require mental time travel. But, it does require remembering to do something in the future, rather than immediately, and then implementing that intention when the time comes. And, as too many of us have learned (with email attachments and other things), even the short timescale and lack of any need for mental time travel does not assure successful completion of these intended actions.

Rather than trying to prove that animals do (or do not) experience mental time travel, the goal of our research that we summarize in this paper is to learn the extent to which nonhuman primates are capable of anticipating a future situation and then controlling their decisions now and in the future so as to best garner rewards. This form of prospective cognition (what Raby and Clayton, 2009, call semantic prospection) does not require seeing yourself, as an "experiencing self," in that future situation (as would episodic future thinking), but it is still an important aspect of human cognition, and one for which comparative data can be informative in understanding human cognition. This research was guided by the literature on human prospective memory.

Prospective memory (PM) consists of the processes of forming intentions, retaining those intentions during a delay period, and properly executing the intended responses when the correct time is reached or correct event is experienced (e.g., Einstein \&
McDaniel, 2005; Ellis, 1996; Kliegel, McDaniel, \& Einstein, 2000; Marsh, Hicks, \& Landau, 1998). Two additional critical features of PM tests are that execution of the intended action cannot occur immediately, and that continuous rehearsal is prevented or minimized (McDaniel \& Einstein, 2007). Although PM is easily disrupted and fragile, adult humans routinely use it as a tool for accomplishing intended future behaviors (Einstein, McDaniel, Manzi, Cochran, \& Baker, 2000; Einstein, McDaniel, Smith, \& Shaw, 1998; Hicks, Marsh, \& Cook, 2005; Kliegel, Mackinlay, \& Jager, 2008; Marsh, Hancock, \& Hicks, 2002; McDaniel, Einstein, Stout, \& Morgan, 2003; Smith, 2003, 2008).

Although good comparative research has been conducted to look at planning abilities in animals and anticipation of future events (e.g., Chappell \& Kacelnik, 2002; Cheke \& Clayton, 2012; Correia, Dickinson, \& Clayton, 2007, Mulcahy \& Call, 2006; Raby, Alexis, Dickinson, \& Clayton, 2007; Volter \& Call, 2014) relatively little research has tried to directly assess human PM-like abilities in animals. A few animal studies have focused on the ability to remember information for future use (sometimes called prospective coding; see Cook, Brown, \& Riley 1985). More recently, evidence for $\mathrm{PM}$ in rats has emerged. For instance, Wilson and Crystal (2012) trained rats to perform a bisection task in which they classified different temporal events as having long or short durations. Rats also learned that after 90 minutes of doing the bisection task they would have 30 minutes of access to food pellets. To gain that food, they had to poke their noses into a food trough, and thus had to stop the bisection task. When working on the bisection task, the rats performed more poorly as the time for the post-test meal approached, and they started making more nose pokes in the food trough, suggesting that they formed a time-based PM to nose-poke around 90 minutes into the bisection task and then implemented that memory a little early. Wilson, Pizzo, and Crystal (2013) gave rats an auditory cue that the access to the big 
meal was forthcoming, and this also produced a decline in performance on the bisection task, suggesting that the rats were anticipating the meal. These two studies showed evidence of a time-based and an event-based prospective memory capacity in rats. Our efforts to contribute to a literature on nonhuman animal PM have focused on nonhuman primates, and in particular by examining how well these animals can anticipate future needs, remember what needs to be done later, and then implement that memory when the time is appropriate.

\section{Prospective Memory in Monkeys}

Our first attempt at examining prospective cognition in monkeys focused on their ability to remember future responses, or what is called prospective coding, in a matching-tosample task (Beran, Evans, Klein, \& Einstein, 2012). Rhesus monkeys and capuchin monkeys performed a computerized task that required encoding a future response at the outset of each trial before the monkeys engaged in a different, ongoing task. Monkeys of both species anticipated what response they would need to make later, during the matching phase of the task, and they made correct responses when the time was right for those responses. However, this task did not capture all of the elements of human PM tasks because monkeys only had to recognize and remember the correct future response that would be needed, but they did not have to spontaneously initiate that response at the appropriate time. Instead, a cue was given by the computer program when it was time to make the delayed response.

To overcome the limitation in the Beran et al. (2012) task, Evans and Beran (2012) presented rhesus monkeys and capuchin monkeys with a joystick task in which the monkeys had to remember a PM cue (a flashing red border on the screen) when it was embedded within an ongoing two-choice discrimination learning set (LS) task. At a break in the ongoing task, monkeys had the opportunity to use the joystick that they controlled with their hands to respond to the task to indicate whether or not they had recently seen the PM cue. Remembering this cue resulted in a large food reward (whereas incorrectly indicating that there was a recent $\mathrm{PM}$ cue when in fact there had not been a cue resulted in a long time-out penalty). Critically, during this response period, the icon representing the PM response was delayed in its appearance relative to the cursor controlled by the monkey's joystick. This allowed an assessment of whether the monkeys would remember to make the PM response prior to receiving a reminder to do so (for more details on this testing method, see Figure 1).

Each block of LS trials consisted of 6 trials with the same $\mathrm{S}+$ (rewarded) and $\mathrm{S}-$ (punished) stimuli being presented in random locations. Sometimes during these 6 trials, the PM cue was given as the perimeter of the screen flashed red. If monkeys later could remember to report they had seen that cue (by moving to an icon on the screen that represented the "cue was seen" response between blocks of trials), they would receive a large food reward. But, falsely reporting that the cue had been present by touching the "cue was seen" response led to a large timeout punishment during which the task was taken away and the monkeys could not work. In the most difficult version of this test, the PM cue would appear during the 5 -second ITI between the first two trials in the block, and so this information had to be retained for the rest of the block before it could be used, and it had to be retained while the monkeys also had to remember the $\mathrm{S}+$ and $\mathrm{S}-$ for the block of trials. And, in these sessions, monkeys were only presented the PM cue in $10 \%$ of the LS blocks. Almost all monkeys reported seeing the PM cue in a statistically significant proportion of trial blocks when it was presented versus when it was not. And, some of the monkeys even anticipated the appearance of the "cue was seen" icon and moved their cursor closer to where that icon would appear even before it did appear. This suggested that even without 


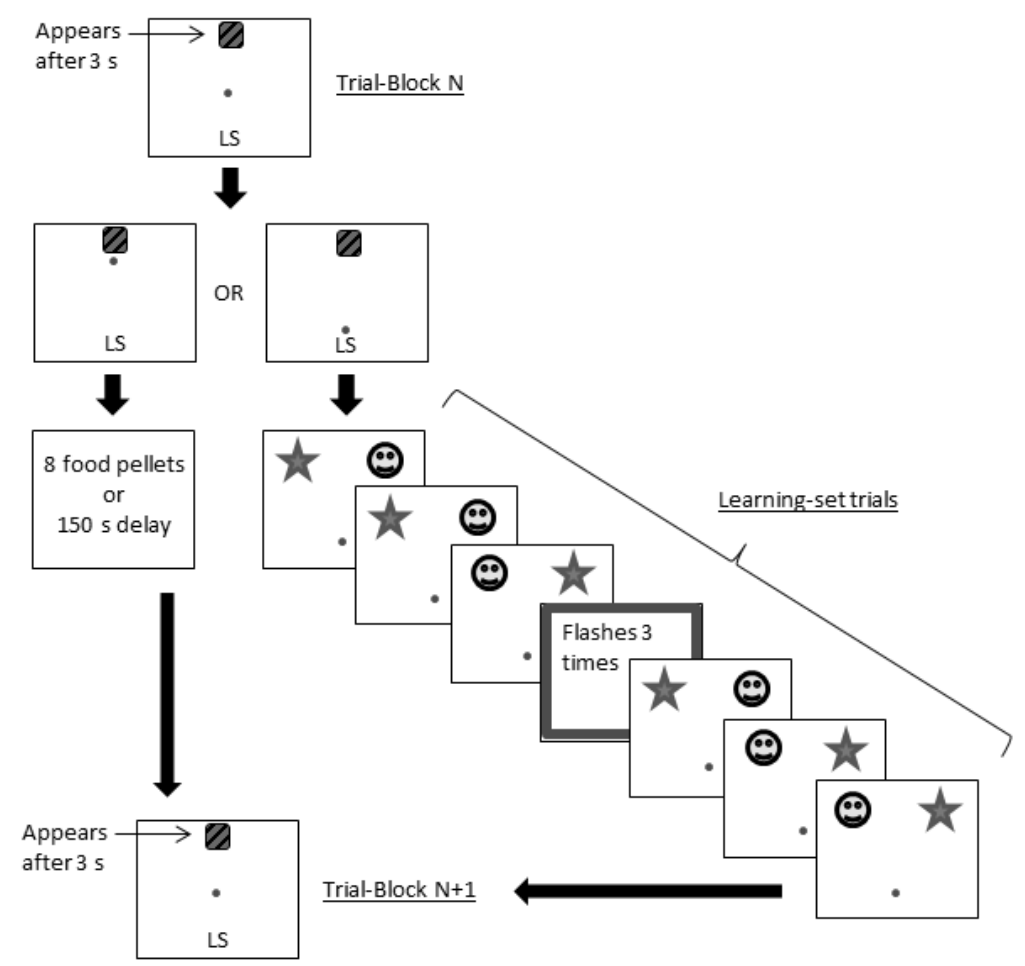

Figure 1. A sample test trial block in the prospective memory task given to monkeys. The diagonally striped square is the prospective memory (PM) response icon and the letters "LS" are the icon for the learning-set task. The small circle is the cursor that monkeys control with the movement of the joystick. Contacting the LS icon with the cursor initiated a set of rewarded LS trials. The PM cue, a flashing thick border, could appear between any two of these trials. Contacting the PM icon resulted in either a large reward or large penalty, depending on whether a PM cue was presented in the previous LS trial-block. From 'Evans, T. A., \& Beran, M. J. (2012). Monkeys exhibit prospective memory in a computerized task. Cognition, 125, 131-140.'

any visual prompts regarding whether they had seen the cue or not, some monkeys responded in anticipation of reporting that cue's presence in the previous trial block. Thus, these monkeys encoded, stored, and retrieved a stimulus event after a delay of at least 45 seconds in this task, and when the occurrence of that event was quite infrequent. Several of these monkeys also anticipated making the PM response after they had encoded the PM cue, which indicated that they could spontaneously remember to act without prompting. And, they did this for a cue that was embedded in another ongoing task, and the cue itself offered no food reward or benefit when it appeared. It was only valuable to remember its appearance (or not) at a later time when the monkeys could use that information to gain a big reward or avoid a long timeout.

\section{Prospective Memory in Chimpanzees}

In our work with chimpanzees, we have been interested in whether an animal model could demonstrate most or all of the components involved in human-like prospective memory tests. In our first study, we tested an experimentally sophisticated chimpanzee named Panzee, who had years of experience in memory tests and a variety of other cognitive tests (Beran \& Beran, 2004; Beran, Smith, \& Perdue, 2012; Evans \& Beran, 2007; Menzel, 1999, 2005; Parrish \& Beran, 2014; Sayers \& Menzel, 2012). We took advantage of Panzee's history of language training and specifically her use and under- 
standing of symbolic tokens. Panzee had considerable experience in relating information about her environment to the humans around her, and she did this through use of the lexigram symbol system, which consists of geometric forms that each represent a different kind of item, including foods, objects, and individuals (Beran \& Heimbauer, 2015; Brakke \& Savage-Rumbaugh, 1995, 1996; Rumbaugh \& Washburn, 2003). For this first study of chimpanzee PM (Beran, Perdue, Bramlett, Menzel, \& Evans, 2012), Panzee was provided with a set of lexigram tokens, which were individual hand-held versions of the symbols Panzee used on her keyboard on a daily basis. Each token was one-sided with a plain white surface on the back side. Panzee had to remember to find, transport, and exchange a specific lexigram token that represented a hidden food item that would be available to her in the future. At the start of each trial, Panzee was presented with two food options to choose between. Panzee then saw the food option that she did not choose being placed into an opaque container outside of her enclosure, and this became the to-be-remembered item for that trial. Panzee's chosen food option was immediately distributed in an adjacent outdoor enclosure along with eight facedown lexigram tokens, one of which matched the hidden food. Panzee was then given immediate access to this enclosure, and while she was outdoors the container holding the originally chosen, and now hidden item, was out of view. Panzee was allowed 30 minutes in the outdoor enclosure to freely forage for food, interact with the tokens, and/or enjoy the surroundings of the enclosure. Meanwhile, an experimenter naïve to the container contents waited indoors and was available to exchange any hidden food item for the correct token. The question was whether Panzee would remember the food type that she had just rejected in favor of another food type when she later had exhausted her foraging for the preferred item. This experiment also included a number of control trials in which there was nothing indoors for her to name after foraging outdoors was complete.

In this experiment, Panzee made significantly more token exchanges during trials with a food item indoors than expected by a random distribution across the two trial types (control and test). Conversely, the absence of a token exchange occurred significantly more often during control trials. Further, on test trials in which Panzee exchanged a token, she presented the correct token significantly more often than predicted by chance. Panzee's behavior in this experiment reflected several of the hallmark features of human prospective memory performance. First, Panzee seemed to prospectively encode the to-be-remembered item. That item was less preferred at the time of choice, and so encoding its identity at that time was the relatively least valuable item to Panzee. Second, Panzee engaged in an unrelated ongoing task between encoding and retrieval that likely occupied much of her motivation and attention given the preference level of her selected foods. In most trials, she also spent additional time surveying the woods outside of the enclosure between foraging for the preferred food and searching for a token. Also, Panzee clearly searched for a specific token in most trials, as she ended her search and immediately returned indoors with the correct token. This represented her retrieval of the prospective memory following the intervening task. Finally, in most cases, Panzee spontaneously implemented the prospective responses of token transport and exchange, and there were no obvious external cues that would have engaged her retrospective memory.

Having demonstrated prospective memory in one highly experienced chimpanzee, we next developed a test that we could present to our full colony of chimpanzees, regardless of testing and rearing history, and also could give a highly similar test to 3-yearold children (Perdue, Beran, Williamson, Gonsiorowski, \& Evans, 2014). The group of four chimpanzees included Panzee and three individuals (Lana, Mercury, and Sherman) with considerably less memory testing experi- 
ence than Panzee, one of which (Mercury) had no history of language training either. The task involved three phases. First, a chimpanzee chose between two food options and received the chosen food for immediate consumption (children chose between two toys). The unchosen, and presumably less valuable, item was then placed under an opaque container that was set away from the test area and out of direct view of the chimpanzee or the child. Next, a new experimenter, who was naïve as to what was in the opaque container, sat in the test area and presented the chimpanzee or child with a series of quantity judgment trials. These trials consisted of the experimenter dropping different numbers of grapes (for chimpanzees) or paper clips (children), one by one, into two opaque containers that the subjects chose between (as in Beran, 2001, 2004). After these quantity trials, the experimenter began removing the test materials. To get the item in the opaque container, the chimpanzee had to get the experimenter's attention and direct them to retrieve the item in that container by using vocalizations, gestures and directional pointing. Children had to ask for the item, and if they failed to do that, they were asked if they were supposed to be remembering anything, and then asked what was in the container.

The chimpanzees remembered to ask for the concealed food item in approximately 85\% of the sessions following the quantity judgment trials. In control trials, with nothing hidden, there was rarely an attempt to retrieve anything. Thus, all four chimpanzees exhibited critical aspects of prospective memory here. At the beginning of the sessions, they formed the intention to get the hidden food item (again, this was for an item that was of lesser value than their chosen item). They then stored that information throughout the time they performed the quantity judgment task, which was a fairly challenging working memory task for these animals. After the quantity judgment task, they retrieved their intention and took action to obtain the hidden item, and this occurred without any cue from the experimenters. Critically, the performance of the chimpanzees in many ways paralleled that of human children who were given a highly similar test, and who also sometimes remembered to retrieve the delayed reward but sometimes forgot.

A large difference between a typical human PM test and the tests outlined to this point is the degree of integration of the PM cue or retrieval opportunity into the concurrent task. A typical PM task given to humans begins with an experimenter instructing the participant to remember to perform a specific act upon the appearance of a particular word or word category on a computer screen (e.g., Einstein et al., 2005; Scullin, McDaniel, \& Einstein, 2010). For example, when the participant sees the target word, he or she is supposed to remember to press a special key on the keyboard. Then, following a delay interval, the participant begins working on an unrelated task. Critically, when the participant sees the target word appear in the unrelated task, he or she needs to remember to press the special key. We presented our version of this common human PM test to the three chimpanzees in our laboratory that have had language training (Evans, Perdue, \& Beran, 2014). The chimpanzees saw a target food item hidden in a tin container at the back of the test area. They then participated, at the front of the enclosure, in a matching task in which they sorted individual lexigram tokens into one of two bowls. Each bowl was attached to a board displaying a set of three photographs, and only one photograph matched each of the presented tokens. Critically, when a chimpanzee received the token that represented the item hidden behind the test area, they needed to remember to pass that token to the experimenter seated by the hidden item (rather than sort it into one of the two bowls) if they wanted to receive the food item represented by that lexigram. So, in essence, they had to remember to name the item at the back of the enclosure later in the trial, when they saw its name as part of the 
ongoing sorting activity at the front of the enclosure.

As in the previous tests, there were occasions in which there was no target item to remember. In these cases, the chimpanzees never attempted to trade a token to the experimenter seated by the concealed item. Conversely, when there was a hidden item in the container, the chimpanzees often remembered to pass a token to the experimenter, although they also sometimes traded out an incorrect lexigram suggesting that this task can be difficult for chimpanzees. One possible shortcoming of this experimental method was that every test session involved a PM target, and this may have overly stressed the PM component of the task relative to the ongoing task component.

Experiment 2 included a new control condition in which there was no prospective memory requirement. In these sessions, the target food item that could be obtained later with a correct lexigram exchange was placed in full view of the chimpanzee under the chair of the experimenter that was conducting the ongoing task trials. The experimenter frequently made sure that the chimp was aware of this item by pointing under the chair before each matching trial. And, as in the test condition with a PM item to be remembered, the chimpanzee could either match a given token to a set of photos, or it could slide the token under the mesh to an experimenter to ask for the visible food item. Evans et al. (2014) assessed whether the chimpanzees would continue to encode, store, retrieve, and execute the PM in test sessions, as they did in Experiment 1, now that they did not always have to remember to do so in all sessions. In trials in which there was no target item available, in either condition, chimpanzees rarely attempted to trade the token. Conversely, in the new control condition in which the target item was visible under the experimenter's chair, the chimpanzees typically passed the token to the experimenter and obtained the item. Similarly, in the test condition, chimpanzees typically passed the token to the experiment- er seated near the hidden food item at the back of the enclosure in order to obtain that item when it was available. Unlike in Experiment 1, when the chimpanzees did pass a token to obtain the target item, it was always the correct token in this experiment. So, in this version of the task in which there was not always a prospective memory requirement, the chimpanzees continued to encode, store, retrieve, and execute a PM when it was necessary to do so.

In Experiment 3, Evans et al. (2014) made the matching trials of the ongoing task more difficult by adding a memory component. After the chimpanzees viewed the token and possible match images for a particular trial, the experimenter flipped over the token so the lexigram could no longer be seen, and he then covered the match images with opaque material for 10 seconds. Thus, the chimpanzees had to remember what items were to be sorted into which bins rather than being allowed to visually match as in the previous experiments. Given this more demanding ongoing task, the question was whether performance would now vary as a function of whether there was a PM requirement (item hidden at back of enclosure) or not (item in plain view at front of enclosure) in a particular session. In the human PM literature, there is interest in determining what degree of monitoring resources individuals devote to remembering to perform a delayed intention (Guynn, 2003; Einstein \& McDaniel, 2005; Marsh et al, 2002; Smith, 2003). This question is often addressed by observing what effect, if any, encoding/ storing a prospective intention has on participants' performance in the ongoing task. Previously, only rats had shown that a disruption in ongoing task performance occurred when PM was engaged during that ongoing task (Wilson \& Crystal, 2012; Wilson, Pizzo, \& Crystal, 2013). However, Evans et al. (2014) found in their Experiment 3 that having to remember to perform the delayed PM response (taking the token to the back of the enclosure) influenced the accuracy of chimpanzees' ongoing matching task perfor- 
mance. This finding suggests that working memory load and prospective memory may have a similar relationship in nonhuman primates. Taken altogether, the results from Evans et al. (2014) demonstrated that chimpanzees have some capacity for human-like prospective memory and can demonstrate such ability in tasks similar in design and complexity to those used with humans.

\section{Summary}

As a whole, this series of prospective memory studies has taught us that nonhuman primates are capable of exhibiting the critical components of prospective memory, as we define it in humans (including encoding, storage, retrieval, and execution). We found that they can sometimes do this when the target item is the less preferred reward available in a particular situation, also, when the task is not based on the vast experimental history of a particularly experienced individual, and, when the PM cue is embedded within other ongoing task activity. However, we have also found that monkeys and chimpanzees are not always successful in these types of tests. They sometimes forget to perform the intended action or they sometimes find creative solutions that do not require memory. Yet, importantly, people do this too. They often forget to do things, and as a result, they rely on strategically placed notes, alarms, and email reminders so they do not necessarily have to remember what they need to do later. So, monkeys' and chimpanzees' performances in PM tests are analogous to human prospective memory, for better or worse.

Of course, this does not mean that nonhuman primate species have the same experiences when they remember to do things later that humans have, nor do these data indicate that the animals must be capable of mental time travel to accomplish what they have in these tests. But, the resulting performance suggests that anticipation of future events and possible outcomes does occur in animals, and they can structure present behavior and can encode and remember potential future responses to meet those future events or outcomes. Continued examinations of such prospective memory capacities in nonhuman animals, and greater understanding of what kinds of things animals can anticipate and perhaps even plan to do will shed light on the emergence of future-oriented cognition in our species. And, those examinations may eventually highlight the similarities and differences in future-oriented processes that are relevant to mental time travel, even if they are not reliant upon that particular construct.

\section{Acknowledgments}

The research summarized in this article was supported by NIH grant HD060563 and by NSF grant BCS0924811. We thank the animal care staff at the Language Research Center for their assistance with this research program and their care of the nonhuman primates.

\section{References}

Atance, C. M., \& O’Neill, D. K. 2001 Episodic future thinking. Trends in Cognitive Sciences, 5, 533-539.

Beran, M. J. 2001 Summation and numerousness judgments of sequentially presented sets of items by chimpanzees (Pan troglodytes). Journal of Comparative Psychology, 115, 181-191.

Beran, M. J. 2004 Chimpanzees (Pan troglodytes) respond to nonvisible sets after one-by-one addition and removal of items. Journal of Comparative Psychology, 118, 25-36.

Beran, M. J., \& Beran, M. M. 2004 Chimpanzees remember the results of one-by-one addition of food items to sets over extended time periods. Psychological Science, 15, 94-99.

Beran, M. J., Evans, T. A., Klein, E. D., \& Einstein, G. O. 2012 Rhesus monkeys (Macaca mulatta) and capuchin monkeys (Cebus apella) remember future responses in a computerized task. Journal of Experi- 
mental Psychology: Animal Behavior Processes, 38, 233-243.

Beran, M. J., \& Heimbauer, L. A. 2015 A longitudinal assessment of vocabulary retention in symbol-competent chimpanzees (Pan troglodytes). PLoS ONE.

Beran, M. J., Perdue, B. M., Bramlett, J. L., Menzel, C. R., \& Evans, T. A. 2012 Prospective memory in a language-trained chimpanzee (Pan troglodytes). Learning and Motivation, 43, 192-199.

Beran, M. J., Smith, J. D., \& Perdue, B. M. 2013 Language-trained chimpanzees name what they have seen, but look first at what they have not seen. Psychological Science, 24, 660-666.

Brakke, K. E., \& Savage-Rumbaugh, E. S. 1995 The development of language skills in bonobo and chimpanzee: I. Comprehension. Language and Communication, 15, 121-148.

Brakke, K. E., \& Savage-Rumbaugh, E. S. 1996 The development of language skills in Pan: II Production. Language and Communication, 16, 361-380.

Chappell, J., \& Kacelnik, A. 2002 Tool selectivity in a non-primate, the New Caladonian crow (Corvus moneduloides). Animal Cognition, 5, 71-78.

Cheke, L. G., \& Clayton, N. S. 2012 Eurasian jays (Garrulus glandarius) overcome their current desires to anticipate two distinct future needs and plan for them appropriately. Biology Letters, 8, 171-175.

Clayton, N. S., Correia, S. P. C., Raby, C. R., Alexis, D. M., Emery, N. J., \& Dickinson, A. 2008 Response to Suddendorf \& Corballis (2008): In defense of animal foresight. Animal Behaviour, 76, e9-e11.

Cook, R. G., Brown, M. F., \& Riley, D. A. 1985 Flexible memory processing by rats: Use of prospective and retrospective information in the radial maze. Journal of Experimental Psychology: Animal Behavior Processes, 11, 453-469.

Correia, S. P. C., Dickinson, A., \& Clayton, N. S. 2007 Western scrub-jays anticipate future needs independently of their current motivational state. Current Biolo- $g y, 17,856-861$

Crystal, J. D. 2013 Remembering the past and planning for the future in rats. Behavioural Processes, 93, 39-49.

Einstein, G. O., \& McDaniel, M. A. 2005 Prospective memory: Multiple retrieval processes. Current Directions in Psychological Science, 14, 286-290.

Einstein, G. O., McDaniel, M. A., Manzi, M., Cochran, B., \& Baker, M. 2000 Prospective memory and aging: Forgetting intentions over short delays. Psychology and Aging, $15,671-683$

Einstein, G. O., McDaniel, M. A., Smith, R. E., \& Shaw, P. 1998 Habitual prospective memory and aging: Remembering intentions and forgetting actions. Psychological Science, 9, 284-288.

Einstein, G. O., McDaniel, M. A., Thomas, R. A., Mayfield, S., Shank, H., et al. 2005 Multiple processes in prospective memory retrieval: Factors determining monitoring versus spontaneous retrieval. Journal of Experimental Psychology: General, 134, 327-342.

Ellis, J. A. 1996 Prospective memory or the realization of delayed intentions: A conceptual framework for research. In M. Brandimonte, G. O. Einstein \& M. A. McDaniel (Eds.), Prospective memory: The ory and applications (pp.1-22). Mahwah, NJ: Lawrence Erlbaum.

Evans, T. A., \& Beran, M. J. 2007 Chimpanzees use self-distraction to cope with impulsivity. Biology Letters, 3, 599-602.

Evans, T. A., \& Beran, M. J. 2012 Monkeys exhibit prospective memory in a computerized task. Cognition, 125, 131-140.

Evans, T. A., Perdue, B. M., \& Beran, M. J. 2014 The relationship between eventbased prospective memory and ongoing task performance in chimpanzees (Pan troglodytes). PLoS ONE, 9, e112015.

Griffiths, D., Dickinson, A., \& Clayton, N. 1999 Episodic memory: What can animals remember about their past? Trends in Cognitive Sciences, 3, 74-80.

Guynn, M. J. 2003 A two-process model of strategic monitoring in event-based 
prospective memory: Activation/retrieval mode and checking. International Journal of Psychology, 38, 245-256.

Hicks, J. L., Marsh, R. L., \& Cook, G. I. 2005 Task interference in time-based, eventbased, and dual intention prospective memory conditions. Journal of Memory and Language, 53, 430-444.

Klein, S. B., Robertson, T. E., \& Delton, A. W. 2010 Facing the future: Memory as an evolved system for planning future acts. Memory and Cognition, 38, 13-22.

Kliegel, M., Mackinlay, R., \& Jager, T. 2008 Complex prospective memory: Development across the lifespan and the role of task interruption. Developmental Psychology, 44, 612-617.

Kliegel, M., McDaniel, M. A., \& Einstein, G. O. 2000 Plan formation, retention, and execution in prospective memory: A new approach and age related effects. Memory \& Cognition, 28, 1041-1049.

Marsh, R. J., Hancock, T. W., \& Hicks, J. L. 2002 The demands of an ongoing activity influence the success of event-based prospective memory. Psychonomic Bulletin and Review, 9, 604-610.

Marsh, R. J., Hicks, J. L., \& Landau, J. D. 1998 An investigation of everyday prospective memory. Memory \& Cognition, 26, 633-643.

McDaniel, M. A., \& Einstein, G. O. 2007 Prospective memory. Los Angeles: Sage Publications.

McDaniel, M. A., Einstein, G. O., Stout, A. C., \& Morgan, Z. 2003 Aging and maintaining intention over delays: Use it or lose it. Psychology and Aging, 18, 807822.

Menzel, C. R. 1999 Unprompted recall and reporting of hidden objects by a chimpanzee (Pan troglodytes) after extended delays. Journal of Comparative Psychology, 113, 426-434.

Menzel, C. R. 2005 Progress in the study of chimpanzee recall and episodic memory. In H. S. Terrace \& J. Metcalfe (Eds.), The missing link in cognition: Origins of self-reflective consciousness (pp.188-224).
New York: Oxford University Press.

Mulcahy, N. J., \& Call, J. 2006, 19 May Apes save tools for future use. Science, 312, 1038-1040.

Parrish, A. E., \& Beran, M. J. 2014 When less is more: Like humans, chimpanzees (Pan troglodytes) misperceive food amounts based on plate size. Animal Cognition, 17, 427-434.

Perdue, B. M., Beran, M. J., Williamson, R. A., Gonsiorowski, A., \& Evans, T. A. 2014 Prospective memory in children and chimpanzees. Animal Cognition, 17, 287295.

Raby, C. R., Alexis, D. M., Dickinson, A., \& Clayton, N. S. 2007 Planning for the future by western scrub-jays. Nature, 445, 919-921.

Raby, C. R., \& Clayton, N. S. 2009 Prospective cognition in animals. Behavioural Processes, 80, 314-324.

Roberts, W. A. 2002 Are animals stuck in time? Psychological Bulletin, 128, 473-489.

Roberts, W. A. 2007 Mental time travel: Animals anticipate the future. Current Biology, 17, 418-420.

Rumbaugh, D. M., \& Washburn, D. A. 2003 Intelligence of apes and other rational beings. New Haven: Yale University Press.

Sayers, K., \& Menzel, C. R. 2012 Memory and foraging theory: chimpanzee utilization of optimality heuristics in the rankorder recovery of hidden foods. Animal Behaviour, 84, 795-803.

Scullin, M. K., McDaniel, M. A., \& Einstein, G. O. 2010 Control of cost in prospective memory: Evidence for spontaneous retrieval processes. Journal of Experimental Psychology: Learning, Memory, \& Cognition, 36, 190-203.

Shettleworth, S. J. 2007 Planning for breakfast. Nature, 445, 825-826.

Smith, R. E. 2003 The cost of remembering to remember in event-based prospective memory: Investigating the capacity demands of delayed intention performance. Journal of Experimental Psychology: Learning, Memory, and Cognition, 29, 347-361. 
BERAN $\cdot$ PERDUE $\cdot$ EVANS : Prospective memory in nonhuman primates

Smith, R. E. 2008 Attention, memory, and delayed intentions. In M. Kliegel, M. A. McDaniel \& G. O. Einstein (Eds.), Prospective memory: Cognitive, neuroscience, developmental and applied perspectives (pp.29-52). New York: Lawrence Erlbaum.

Suddendorf, T., \& Busby, J. 2003 Mental time travel in animals? Trends in Cognitive Sciences, 7, 391-396.

Suddendorf, T., \& Corballis, M. C. 2007 The evolution of foresight: What is mental time travel, and is it unique to humans? Behavioral and Brain Sciences, 30, 299-351.

Tulving, E. 2005 Episodic memory and autonoesis: Uniquely human? In H. Terrace \& J. Metcalfe (Eds.), The missing link in cognition: Evolution of self-knowing con sciousness (pp.3-56). New York: Oxford University Press.

Volter, C. J., \& Call, J. 2014 Younger apes and human children plan their moves in a maze task. Cognition, 130, 186-203.

Wilson, A. G., \& Crystal, J. D. 2012 Prospective memory in the rat. Animal Cognition, 15, 349-358.

Wilson, A. G., Pizzo, M. J., \& Crystal, J. D. 2013 Event-based prospective memory in the rat. Current Biology, 23, 1089-1093.

Zentall, T. R. 2006 Mental time travel in animals: A challenging question. Behav ioural Processes, 72, 173-183.

(2015. 2.17 受稿, 2015. 3.25 受理) 